\begin{tabular}{|c|c|}
\hline Title & Quantum mechanical grad-B drift velocity operator in a weakly non-uniform magnetic field \\
\hline Author(s) & Chan, Poh Kam; Oikawa, Shun-ichi; Kosaka, W ataru \\
\hline Citation & $\begin{array}{l}\text { Phy sics of Plasmas, 23(2), } 022104 \\
\text { https://doi.org/10.1063/1.4941096 }\end{array}$ \\
\hline Issue Date & 2016-02-05 \\
\hline Doc URL & http:/hdl.handle.net/2115/64482 \\
\hline Rights & $\begin{array}{l}\text { Copyright } 2016 \text { A merican Institute of Physics. This article may be downloaded for personal use only. A ny other use } \\
\text { requires prior permission of the author and the A merican Institute of Phy sics. The following article appeared in Phys. } \\
\text { Plasmas } 23,022104 \text { (2016) and may be found at } \\
\text { http://scitation.aip.org/content/aip/pournal/pop/23/2/10.1063/1.4941096. }\end{array}$ \\
\hline Type & article \\
\hline File Information & 1.4941096.pdf \\
\hline
\end{tabular}

Instructions for use 


\section{AIP | Physics of \\ Plasmas}

\section{Quantum mechanical grad-B drift velocity operator in a weakly non-uniform magnetic field}

Poh Kam Chan, Shun-ichi Oikawa, and Wataru Kosaka

Citation: Physics of Plasmas 23, 022104 (2016); doi: 10.1063/1.4941096

View online: http://dx.doi.org/10.1063/1.4941096

View Table of Contents: http://scitation.aip.org/content/aip/journal/pop/23/2?ver=pdfcov

Published by the AIP Publishing

\section{Articles you may be interested in}

Effects of magnetic field on the interaction between terahertz wave and non-uniform plasma slab

Phys. Plasmas 22, 103302 (2015); 10.1063/1.4931742

Formation of current filaments and magnetic field generation in a quantum current-carrying plasma

Phys. Plasmas 20, 092310 (2013); 10.1063/1.4823725

Effects of the non-uniform initial environment and the guide field on the plasmoid instability

Phys. Plasmas 20, 061206 (2013); 10.1063/1.4811144

The parametric decay of dust ion acoustic waves in non-uniform quantum dusty magnetoplasmas

Phys. Plasmas 18, 063705 (2011); 10.1063/1.3595235

Electrostatic drift waves in nonuniform quantum magnetized plasmas

Phys. Plasmas 15, 082103 (2008); 10.1063/1.2967479

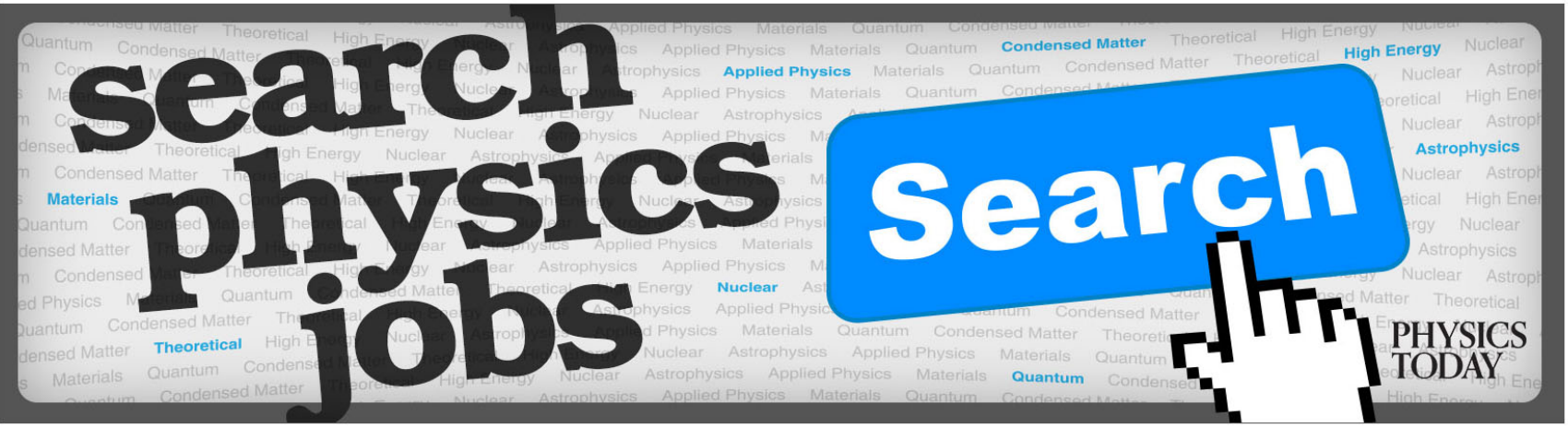




\title{
Quantum mechanical grad-B drift velocity operator in a weakly non-uniform magnetic field
}

\author{
Poh Kam Chan, ${ }^{1}$ Shun-ichi Oikawa ${ }^{2}$ and Wataru Kosaka ${ }^{1}$ \\ ${ }^{1}$ Graduate School of Engineering, Hokkaido University, Sapporo 060-8628, Japan \\ ${ }^{2}$ Faculty of Engineering, Hokkaido University, Sapporo 060-8628, Japan
}

(Received 10 December 2015; accepted 19 January 2016; published online 5 February 2016)

This paper presents the analytical solution for quantum mechanical grad- $B$ drift velocity operator by solving the Heisenberg equation of motion. Using the time dependent operators, it is shown the analytical solution of the position operators in $\hat{x}(t)$ and $\hat{y}(t)$ of the particle in the presence of a weakly non-uniform magnetic field. It is also shown numerically that the grad- $B$ drift velocity operator agrees with the classical counterpart. (C) 2016 AIP Publishing LLC.

[http://dx.doi.org/10.1063/1.4941096]

\section{INTRODUCTION}

In the field of plasma physics, grad- $B$ drift velocity is a well-known topic. The gyration of the particle or drift motion of a charged particle has gained the interest of many researchers especially after Alfvén ${ }^{1}$ and Spitzer $^{2}$ had obtained the expression for the drift velocity of a charged particle in the presence of a non-uniform magnetic field. Following that, analytical analysis is getting attention by researchers. Seymour, ${ }^{3,4}$ Hurley, ${ }^{5}$ and Karlson, ${ }^{6}$ obtained the classical particle drift in a static magnetic field using different analytical approaches. Different approaches are being studied by researchers, e.g., Birmingham ${ }^{7}$ uses bounceaverage guiding-center trajectory to solve the drift motion of a charged particle. However, in later research, the drift velocity of a charged particle was studied in a wide field of plasma by using the classical approach. ${ }^{8-14}$

In recent years, the motion of charged particles is getting attention not only in the classical approach but also in the quantum approach. ${ }^{15-20}$ In considering the diffusion of plasmas, it was pointed out more than half a century $\operatorname{ago}^{21,22}$ that the wave character of a charged particle should be taken into consideration when the temperature is high, i.e., when the relative speed of the interacting particle is fast. The criterion required for the classical theory to be valid in terms of relative speed $g$ in hydrogen plasma is given as $g<2 e^{2} /\left(4 \pi \varepsilon_{0} \hbar\right)=4.4 \times 10^{6} \mathrm{~m} /$ $\mathrm{s},{ }^{22}$ where $e$ and $\hbar \equiv h / 2 \pi$ stand for the elementary electric charge and the reduced Planck's constant.

As pointed out in Refs. 23-25 that (i) for distant encounters in the plasma of a temperature $T \sim 10 \mathrm{keV}$ and $n=10^{20} \mathrm{~m}^{-3}$, the average potential energy $U \sim 30 \mathrm{meV}$ is as small as the uncertainty in energy $\Delta E \sim 40 \mathrm{meV}$, and (ii) for a magnetic field $B \sim 3 \mathrm{~T}$, the spatial size of the wavefunction in the plane perpendicular to the magnetic field is as large as the magnetic length $\sigma_{B}=\sqrt{\hbar / e B} \sim 2 \times 10^{-8} \mathrm{~m},{ }^{26}$ which is larger than the typical electron wavelength $\lambda_{e} \sim 10^{-11} \mathrm{~m}$ and is around one-tenth of the average interparticle separation $n^{-1 / 3} \sim 2 \times 10^{-7} \mathrm{~m}$. Thus, for plasma with temperature $T \sim 10 \mathrm{keV}$ or higher, ions as well as electrons should be treated quantum mechanically. In current plasma physics, the quantum mechanical effect enters as a minor correction to the Coulomb logarithm in the case of close encounter. ${ }^{27}$ Nonetheless, the neoclassical theory ${ }^{28}$ is capable of predicting a lot of phenomena such as related to the current conduction.

Such phenomena linearly depend on the change in momentum $\Delta \boldsymbol{p}=m \Delta \boldsymbol{v}$ or in position $\Delta \boldsymbol{r}$ due to the Coulomb interaction. The diffusion, however, is a quadratic function change, such as $\Delta p^{2}$ and $\Delta r^{2}$, and is not properly accounted for in the existing classical and neoclassical theories. Corresponding to these facts, the authors conducted the quantum mechanical analyse ${ }^{29-33}$ on a single charged particle in the presence of external electromagnetic fields, focusing especially on the time development of variance in position and momentum. For the plasma mentioned above, the deviation $\sigma_{r}(t)$ of the ions would reach the interparticle separation $n^{-1 / 3}$ in a time interval of the order of $10^{-4} \mathrm{~s}$. After this time, the wavefunctions of neighboring particles would overlap, as a result the conventional classical analysis may lose its validity. Plasmas may behave like extremelylow-density liquids, not gases, since the size $\sigma_{r}$ of each particle is of the same order of the interparticle separation $n^{-1 / 3}$.

In this paper, it is shown the derivation of drift velocity of a particle in the presence of a non-uniform magnetic field, Landau gauge-like quadratic vector potential $\boldsymbol{A}=-B \hat{y}\left(1-\hat{y} / 2 L_{B}\right) \boldsymbol{e}_{x}$, where $L_{B}$ is the magnetic gradient scale length and $B$ is the magnetic flux density. Here, the quantum mechanical grad- $B$ drift velocity operator is obtained and confirmed with the numerical calculation. More importantly, the solution agrees with the classical drift counterpart.

\section{TIME DEPENDENT OPERATOR IN A UNIFORM MAGNETIC FIELD}

The two-dimensional Schrödinger equation is solved numerically ${ }^{29-33}$ and theoretically for a wavefunction $\psi$ at position $\boldsymbol{r}$ and time $t$

$$
\mathrm{i} \hbar \frac{\partial \psi}{\partial t}=\left\{\frac{1}{2 m}(-\mathrm{i} \hbar \nabla-q \mathbf{A})^{2}+q V\right\} \psi,
$$

where $V$ and A stand for the scalar and vector potentials, $m$ and $q$ are the mass and electric charge of the particle under 
consideration, and $\mathrm{i} \equiv \sqrt{-1}$ is the imaginary unit. In the presence of a uniform magnetic field, with a Landau gauge, ${ }^{26}$ of $A_{x}=-B y, A_{y}=0, A_{z}=0$, and by solving the Heisenberg equation of motion

$$
\mathrm{i} \hbar \frac{\mathrm{d} \hat{X}(t)}{\mathrm{d} t}=[\hat{X}(t), \hat{H}]
$$

where the square bracket $[\cdot, \cdot]$ is the commutator, $\hat{H}$ is the Hamiltonian, $\hat{X}$ can be any operator and its time development $\hat{X}(t)$, it is well known that the time dependent operators for position $\hat{x}(t)$ and $\hat{y}(t)$ are obtained analytically as

$$
\begin{gathered}
\hat{x}(t)=\hat{x}+\frac{\hat{P}_{y}}{q B}-\frac{\hat{P}_{y}}{q B} \cos \omega t+\left(\hat{y}+\frac{\hat{P}_{x}}{q B}\right) \sin \omega t, \\
\hat{y}(t)=-\frac{\hat{P}_{x}}{q B}+\left(\hat{y}+\frac{\hat{P}_{x}}{q B}\right) \cos \omega t+\frac{\hat{P}_{y}}{q B} \sin \omega t,
\end{gathered}
$$

where $\omega \equiv q B / m$ is the cyclotron frequency, $\hat{P}_{y}=-\mathrm{i} \hbar \partial_{y}$ is the $y$-component of the momentum operator, and $\hat{P}_{x}=-\mathrm{i} \hbar \partial_{x}$ is the $x$-component of the momentum operator. The expectation value of the position $\hat{x}(t)$ and $\hat{y}(t)$ is obtained by implementing the initial condition for wavefunction at $\boldsymbol{r}=\boldsymbol{r}_{0}$ with $\boldsymbol{r}_{0}$ being the initial center of wavefunction $\psi$ is given by

$$
\psi(\boldsymbol{r}, 0)=\frac{1}{\sqrt{\pi} \sigma_{B}} \exp \left\{-\frac{\left(\boldsymbol{r}-\boldsymbol{r}_{0}\right)^{2}}{2 \sigma_{B}^{2}}+\mathrm{i} \boldsymbol{k}_{0} \cdot \boldsymbol{r}\right\}
$$

where the magnetic length $\sigma_{B}$ is the initial standard deviation and $\hbar \boldsymbol{k}_{0}$ is the initial canonical momentum.

A particle in a uniform magnetic field can be solved straightforwardly; however, for the non-uniform magnetic case $\boldsymbol{B}=B\left(1-\hat{y} / L_{B}\right) \boldsymbol{e}_{z}$, the theoretical derivations become long and complicated. Spitzer ${ }^{2}$ pointed out that for a general classical equation of drift velocity, it can only be solved analytically by an approximate theory. Hence, in this paper, we solved the Heisenberg equation of motion analytically with the magnetic gradient scale length to first order in $L_{B}^{-1}$. In Section V, both the classical and quantum mechanical grad$B$ drift velocities are obtained.

The grad- $B$ drift velocity analytical solution is compared with the numerical calculation. For the numerical calculation, a two-dimensional Schrödinger equation code is developed and the calculation is done on a GPU (Nvidia GTX-580: 512cores/3GB@1.54 GHz), using CUDA. ${ }^{34}$ Furthermore, the numerical errors had been removed from the numerical calculation by subtracting the variances in the non-uniform magnetic field from the uniform magnetic field. ${ }^{29,30}$

\section{TIME DEPENDENT OPERATOR FOR WEAKLY NON-UNIFORM MAGNETIC FIELD}

In the presence of a weakly non-uniform magnetic field $\boldsymbol{B}=B\left(1-\hat{y} / L_{B}\right) \boldsymbol{e}_{z}$, a Landau gauge-like quadratic vector potential is given as

$$
\boldsymbol{A}=-B \hat{y}\left(1-\frac{\hat{y}}{2 L_{B}}\right) \boldsymbol{e}_{x}
$$

Substituting the vector potential in Eq. (6) into the twodimensional Schrödinger equation in Eq. (1), the Hamiltonian $\hat{H}$ for a charge particle with a mass $m$ and a charge $q$ in the absence of an electrostatic potential for the non-relativistic charge particle is given to first order in $L_{B}^{-1}$ as

$$
\hat{H}=\frac{1}{2 m}\left(\hat{P}_{y}^{2}+\hat{\Pi}_{x}^{2}-\frac{\hat{\Pi}_{x}^{3}}{q B L_{B}}\right),
$$

where

$$
\hat{\Pi}_{x}=\left(1+\frac{\hat{P}_{x}}{q B L_{B}}\right) q B \hat{y}+\left(1+\frac{\hat{P}_{x}}{2 q B L_{B}}\right) \hat{P}_{x} .
$$

The exact mechanical momentum operator $m \hat{\boldsymbol{v}}=\hat{\boldsymbol{P}}-q \hat{\boldsymbol{A}}$ $=(m \hat{u}, m \hat{v})$ is given as

$$
\begin{gathered}
m \hat{u}=\hat{P}_{x}+q B \hat{y}\left(1-\frac{\hat{y}}{2 L_{B}}\right), \\
m \hat{v}=\hat{P}_{y},
\end{gathered}
$$

where $\hat{\boldsymbol{v}}$ is the velocity operator. The mechanical momentum operator $m \hat{u}$ to first order in $L_{B}^{-1}$ is given as

$$
m \hat{u}=\hat{\Pi}_{x}-\frac{\hat{\Pi}_{x}^{2}}{2 q B L_{B}} .
$$

From the Heisenberg equation of motion, the time derivative of $\hat{\Pi}_{x}$ is given as

$$
\frac{\mathrm{d} \hat{\Pi}_{x}}{\mathrm{~d} t}=\left(1+\frac{\hat{P}_{x}}{q B L_{B}}\right) \omega \hat{P}_{y}=\hat{\Omega} \hat{P}_{y}
$$

which leads to the definition of the angular frequency operator as

$$
\hat{\Omega}=\left(1+\frac{\hat{P}_{x}}{q B L_{B}}\right) \omega
$$

On the other hand, for the momentum operator in the $y$-direction $\hat{P}_{y}$, we have

$$
\frac{\mathrm{d} \hat{P}_{y}}{\mathrm{~d} t}=-\hat{\Omega}\left(\hat{\Pi}_{x}-\frac{3 \hat{\Pi}_{x}^{2}}{2 q B L_{B}}\right) .
$$

In order to derive the time dependent momentum operators $\hat{P}_{x}(t)$ and $\hat{\Pi}_{x}(t)$ with Heisenberg equation of motion, Eqs. (11) and (13) are expanded using the Heisenberg picture

$$
\hat{X}(t)=\exp \left(-\frac{t}{\mathrm{i} \hbar} \hat{H}\right) \hat{X} \exp \left(\frac{t}{\mathrm{i} \hbar} \hat{H}\right) .
$$

Let us choose the operator $\hat{X}$ as

$$
\hat{X}=\hat{P}_{x}
$$

and 


$$
\hat{X}=\hat{\Pi}_{x}+\frac{6 \hat{P}_{y}^{2}-9 \hat{\Pi}_{x}^{2}}{2 q B L_{B}} .
$$

Using Heisenberg equation of motion Eq. (2), the time derivative of the operator $\hat{X}$ given in Eq. (16) can be obtained as

$$
\begin{gathered}
\frac{\mathrm{d} \hat{X}}{\mathrm{~d} t}=+\hat{\Omega}^{1}\left(\hat{P}_{y}+\frac{1}{2} \frac{\hat{P}_{y} \hat{\Pi}_{x}+\hat{\Pi}_{x} \hat{P}_{y}}{q B L_{B}}\right)-4(2 \hat{\Omega})^{1} \frac{\hat{\Pi}_{x} \hat{P}_{y}+\hat{P}_{y} \hat{\Pi}_{x}}{q B L_{B}}, \\
\frac{\mathrm{d}^{2} \hat{X}}{\mathrm{~d} t^{2}}=-\hat{\Omega}^{2}\left(\hat{\Pi}_{x}-\frac{3}{2} \frac{\hat{\Pi}_{x}^{2}}{q B L_{B}}-\frac{\hat{P}_{y}^{2}-\hat{\Pi}_{x}^{2}}{q B L_{B}}\right)-4(2 \hat{\Omega})^{2} \frac{\hat{P}_{y}^{2}-\hat{\Pi}_{x}^{2}}{q B L_{B}}, \\
\frac{\mathrm{d}^{3} \hat{X}}{\mathrm{~d} t^{3}}=-\hat{\Omega}^{3}\left(\hat{P}_{y}+\frac{1}{2} \frac{\hat{P}_{y} \hat{\Pi}_{x}+\hat{\Pi}_{x} \hat{P}_{y}}{q B L_{B}}\right)+4(2 \hat{\Omega})^{3} \frac{\hat{\Pi}_{x} \hat{P}_{y}+\hat{P}_{y} \hat{\Pi}_{x}}{q B L_{B}}, \\
\frac{\mathrm{d}^{4} \hat{X}}{\mathrm{~d} t^{4}}=+\hat{\Omega}^{4}\left(\hat{\Pi}_{x}-\frac{3}{2} \frac{\hat{\Pi}_{x}^{2}}{q B L_{B}}-\frac{\hat{P}_{y}^{2}-\hat{\Pi}_{x}^{2}}{q B L_{B}}\right)+4(2 \hat{\Omega})^{4} \frac{\hat{P}_{y}^{2}-\hat{\Pi}_{x}^{2}}{q B L_{B}},
\end{gathered}
$$

and so on. The derivations of time derivative of for operator $\hat{X}$ of the higher orders are obtained and combined together using the Heisenberg picture. Later on, the expressions are simplified by using Taylor expansion and the time derivative for operator $\hat{X}(t)$ obtained as

$$
\hat{X}(t)=\left(\hat{\Pi}_{x}-\frac{3}{2} \frac{\hat{\Pi}_{x}^{2}}{q B L_{B}}-\frac{\hat{P}_{y}^{2}-\hat{\Pi}_{x}^{2}}{q B L_{B}}\right) \cos \hat{\Omega} t+\left(\hat{P}_{y}+\frac{1}{2} \frac{\hat{P}_{y} \hat{\Pi}_{x}+\hat{\Pi}_{x} \hat{P}_{y}}{q B L_{B}}\right) \sin \hat{\Omega} t+4 \frac{\hat{P}_{y}^{2}-\hat{\Pi}_{x}^{2}}{q B L_{B}} \cos 2 \hat{\Omega} t-4 \frac{\hat{\Pi}_{x} \hat{P}_{y}+\hat{P}_{y} \hat{\Pi}_{x}}{q B L_{B}} \sin 2 \hat{\Omega} t
$$

Substituting back the expression for the time derivative for operator $\hat{X}(t)$ into Eq. (16), and the expression for time dependent operators $\hat{P}_{x}(t)$ and $\hat{\Pi}_{x}(t)$, to first order in $L_{B}^{-1}$, are

$$
\begin{gathered}
\hat{P}_{x}(t)=\hat{P}_{x}, \\
\hat{\Pi}_{x}(t)=\frac{3 \hat{P}_{y}^{2}+3 \hat{\Pi}_{x}^{2}}{4 q B L_{B}}+\left(\hat{\Pi}_{x}-\frac{2 \hat{P}_{y}^{2}+\hat{\Pi}_{x}^{2}}{2 q B L_{B}}\right) \cos \hat{\Omega} t+\left(\hat{P}_{y}+\frac{\hat{P}_{y} \hat{\Pi}_{x}+\hat{\Pi}_{x} \hat{P}_{y}}{2 q B L_{B}}\right) \sin \hat{\Omega} t+\frac{\hat{P}_{y}^{2}-\hat{\Pi}_{x}^{2}}{4 q B L_{B}} \cos 2 \hat{\Omega} t-\frac{\hat{\Pi}_{x} \hat{P}_{y}+\hat{P}_{y} \hat{\Pi}_{x}}{4 q B L_{B}} \sin 2 \hat{\Omega} t .
\end{gathered}
$$

Note that the operator $\hat{P}_{x}(t)=\hat{P}_{x}$ does not change with time, since the Hamiltonian $\hat{H}$ in Eq. (7) does not include the position operator $\hat{x}$.

Using the results above, the time dependent operator of position $\hat{x}(t)$ and $\hat{y}(t)$ is obtained. From operator $\hat{\Pi}_{x}$ Eq. (8), the time dependent operator $\hat{y}(t)$ is found and shown, to first order in $L_{B}^{-1}$ as

$$
q B \hat{y}(t)=\left(1-\frac{\hat{P}_{x}}{q B L_{B}}\right) \hat{\Pi}_{x}(t)-\left(1-\frac{\hat{P}_{x}}{2 q B L_{B}}\right) \hat{P}_{x}
$$

which leads to

$$
\begin{aligned}
y(t)= & -\left(\frac{1}{q B}-\frac{\hat{P}_{x}}{2 q^{2} B^{2} L_{B}}\right) \hat{P}_{x}+\frac{3 \hat{P}_{y}^{2}+3 \hat{\Pi}_{x}^{2}}{4 q^{2} B^{2} L_{B}}+\left(\frac{\hat{\Pi}_{x}}{q B}-\frac{2 \hat{P}_{y}^{2}+\hat{\Pi}_{x}^{2}}{2 q^{2} B^{2} L_{B}}-\frac{\hat{P}_{x} \hat{\Pi}_{x}}{q^{2} B^{2} L_{B}}\right) \cos \hat{\Omega} t \\
& +\left(\frac{\hat{P}_{y}}{q B}+\frac{\hat{P}_{y} \hat{\Pi}_{x}+\hat{\Pi}_{x} \hat{P}_{y}}{2 q^{2} B^{2} L_{B}}-\frac{\hat{P}_{x} \hat{P}_{y}}{q^{2} B^{2} L_{B}}\right) \sin \hat{\Omega} t+\frac{\hat{P}_{y}^{2}-\hat{\Pi}_{x}^{2}}{4 q^{2} B^{2} L_{B}} \cos 2 \hat{\Omega} t-\frac{\hat{\Pi}_{x} \hat{P}_{y}+\hat{P}_{y} \hat{\Pi}_{x}}{4 q^{2} B^{2} L_{B}} \sin 2 \hat{\Omega} t .
\end{aligned}
$$

On the other hand, the time dependent operator $\hat{x}(t)$ is obtained by integrating $\hat{u}(t)$ form Eq. (9b) with time $t$

which leads to

$$
\hat{x}(t)=\hat{x}+\int_{0}^{t} \hat{u}(t) \mathrm{d} t
$$




$$
\begin{aligned}
\hat{x}(t)= & \hat{x}+\left(\frac{1}{q B}-\frac{\hat{P}_{x}}{q^{2} B^{2} L_{B}}\right) \hat{P}_{y}+\frac{\hat{\Pi}_{x} \hat{P}_{y}+\hat{P}_{y} \hat{\Pi}_{x}}{4 q^{2} B^{2} L_{B}}+\frac{\hat{P}_{y}^{2}+\hat{\Pi}_{x}^{2}}{2 m q B L_{B}} t-\left(\frac{\hat{P}_{y}}{q B}+\frac{\hat{P}_{y} \hat{\Pi}_{x}+\hat{\Pi}_{x} \hat{P}_{y}}{2 q^{2} B^{2} L_{B}}\right) \cos \hat{\Omega} t \\
& +\left(\frac{\hat{\Pi}_{x}}{q B}-\frac{2 \hat{P}_{y}^{2}+\hat{\Pi}_{x}^{2}}{2 q^{2} B^{2} L_{B}}\right) \sin \hat{\Omega} t+\frac{\hat{\Pi}_{x} \hat{P}_{y}+\hat{P}_{y} \hat{\Pi}_{x}}{4 q^{2} B^{2} L_{B}} \cos 2 \hat{\Omega} t+\frac{\hat{P}_{y}^{2}-\hat{\Pi}_{x}^{2}}{4 q^{2} B^{2} L_{B}} \sin 2 \hat{\Omega} t .
\end{aligned}
$$

From the operator $m \hat{u}$ in Eqs. (9), together with the operator $\hat{\Pi}_{x}(t)$ in Eq. (20), the time dependent momentum operator $m \hat{u}(t)$ along $x$-axis is obtained as

$$
m \hat{u}(t)=\frac{\hat{P}_{y}^{2}+\hat{\Pi}_{x}^{2}}{2 q B L_{B}}+\left(\hat{\Pi}_{x}-\frac{2 \hat{P}_{y}^{2}+\hat{\Pi}_{x}^{2}}{2 q B L_{B}}\right) \cos \hat{\Omega} t+\left(\hat{P}_{y}+\frac{\hat{P}_{y} \hat{\Pi}_{x}+\hat{\Pi}_{x} \hat{P}_{y}}{2 q B L_{B}}\right) \sin \hat{\Omega} t+\frac{\hat{P}_{y}^{2}-\hat{\Pi}_{x}^{2}}{2 q B L_{B}} \cos 2 \hat{\Omega} t-\frac{\hat{\Pi}_{x} \hat{P}_{y}+\hat{P}_{y} \hat{\Pi}_{x}}{2 q B L_{B}} \sin 2 \hat{\Omega} t .
$$

Since the time dependent momentum operator $m \hat{u}(t)$ is obtained, the grad- $B$ drift operator is obtained straightforwardly as

$$
\hat{u}_{\nabla B} \equiv \overline{\hat{u}(t)}=\frac{\hat{P}_{y}^{2}+\hat{\Pi}_{x}^{2}}{2 m q B L_{B}}=\frac{\hat{H}}{q B L_{B}}+O\left(L_{B}^{-2}\right) .
$$

The expectation value of the grad- $B$ drift velocity operator $u_{\nabla B} \equiv\left\langle\hat{u}_{\nabla B}\right\rangle$ is given as follows:

$$
\begin{aligned}
u_{\nabla B} & =\frac{1}{\pi \sigma_{B}^{2}} \int_{\Sigma} e^{-\frac{\left(r-r_{0}\right)^{2}}{2 \sigma_{B}^{2}}-\mathrm{i} \boldsymbol{k}_{0} \cdot \boldsymbol{r}} \hat{u}_{\nabla B} e^{-\frac{\left(r-r_{0}\right)^{2}}{2 \sigma_{B}^{2}}+\mathrm{i} \boldsymbol{k}_{0} \cdot \boldsymbol{r}} \mathrm{d}^{2} \boldsymbol{r} \\
& =\frac{m v_{0}^{2}}{2 q B L_{B}}+\frac{1}{2 q B L_{B}}\left(\frac{\hbar^{2}}{m \sigma_{B}^{2}}+\frac{m \omega^{2} \sigma_{B}^{2}}{2}\right),
\end{aligned}
$$

where $m v_{0} \equiv\langle m \hat{v}\rangle=\langle\hat{P}-q \hat{A}\rangle$. When we use the magnetic length of $\sqrt{\hbar / q B}$ as $\sigma_{B},{ }^{26}$ then we have

$$
u_{\nabla B}=\frac{m v_{0}^{2}}{2 q B L_{B}}+\frac{3 \hbar}{4 m L_{B}} .
$$

Note that the first term of $u_{\nabla B}$ coincides with the classical formula for the grad- $B$ drift, and the second term represents the quantum mechanical drift due to the uncertainty.

\section{NUMERICAL CONFIRMATION}

In order to confirm the theoretical grad- $B$ drift velocity Eq. (28), the numerical calculation is performed and the results are shown in Fig. 1. Numerical grad- $B$ drift velocity is obtained by subtracting the position of the particle in the $x$-direction of a non-uniform magnetic field case from the uniform magnetic case. The subtracted value is the drifted position of the particle in the presence of a non-uniform magnetic field. Note that the constant $(1+\varepsilon)$ from Eq. (29) is the gradient of the increment of the graph in Fig. 1 with $\varepsilon$ being the numerical error.

In these numerical calculations, the parameters are normalized as mass of the particle $m=1.6722 \times 10^{-27} \mathrm{~kg}$, charge $q=1.602 \times 10^{-19} \mathrm{C}$, magnetic flux density $B=10 \mathrm{~T}$, velocity $\bar{v}=10 \mathrm{~m} / \mathrm{s}$, length $\bar{\rho}=1.04382 \times 10^{-8} \mathrm{~m}$, and time $\bar{t}=1.04382 \times 10^{-9} \mathrm{~s}$. Lengths are normalized by cyclotron radius of a proton with a speed of $10 \mathrm{~m} / \mathrm{s}$ in a magnetic field of $10 \mathrm{~T}$. The cyclotron frequency in such a case is used for normalization of the time. This normalization leads to the normalized initial standard deviation given as $\sigma_{B}=\sqrt{\hbar / e B}$ $\cong 0.777$. Let us make the affirmation between theoretical derivation and numerical results by using the deviation analysis below

$$
u_{\nabla B}=(1+\varepsilon)\left(\frac{m v_{0}^{2}}{2 q B L_{B}}+\frac{3 \hbar}{4 m L_{B}}\right)
$$

where $\varepsilon$ is the numerical error. For analytical solution, since it is exact, the numerical error $\varepsilon=0$ and we will get the exact solution as in Eq. (28). Comparisons between analytical solutions with numerical calculation are conducted. With various combinations of physical parameters, such as $m, q$, $v_{0}, B$, and $L_{B}$, the numerical results of grad- $B$ drift velocity are shown in Fig. 2 and the expression is shown in Eq. (30)

$$
u_{\nabla B}=1.0044\left(\frac{m v_{0}^{2}}{2 q B L_{B}}+\frac{3 \hbar}{4 m L_{B}}\right) .
$$

Note that Fig. 2 is the combination of 8 sets of data in Fig. 1.

Comparing between the numerical results Eq. (30) and analytical solutions Eq. (28), it is concluded that the

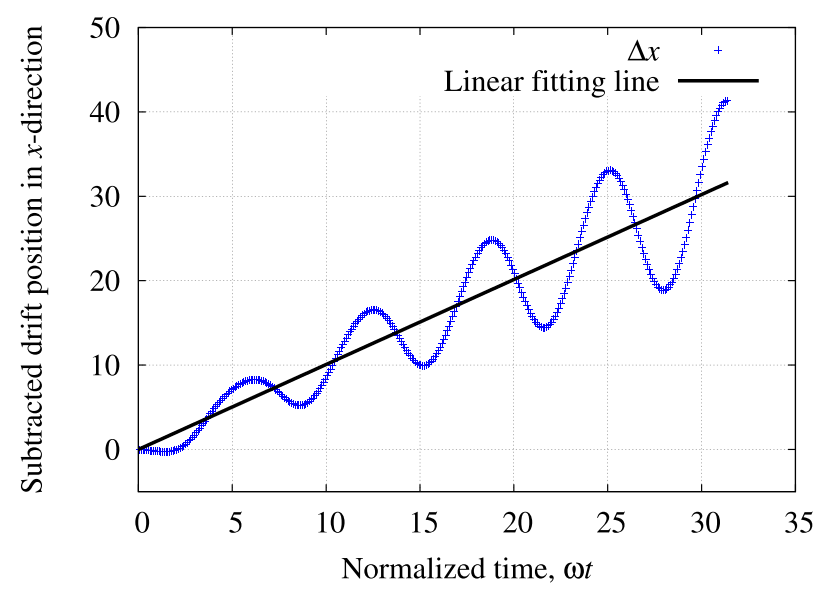

FIG. 1. Numerical time evolution of particle drift in $x$-direction $\Delta x$ for 5 gyrations. 


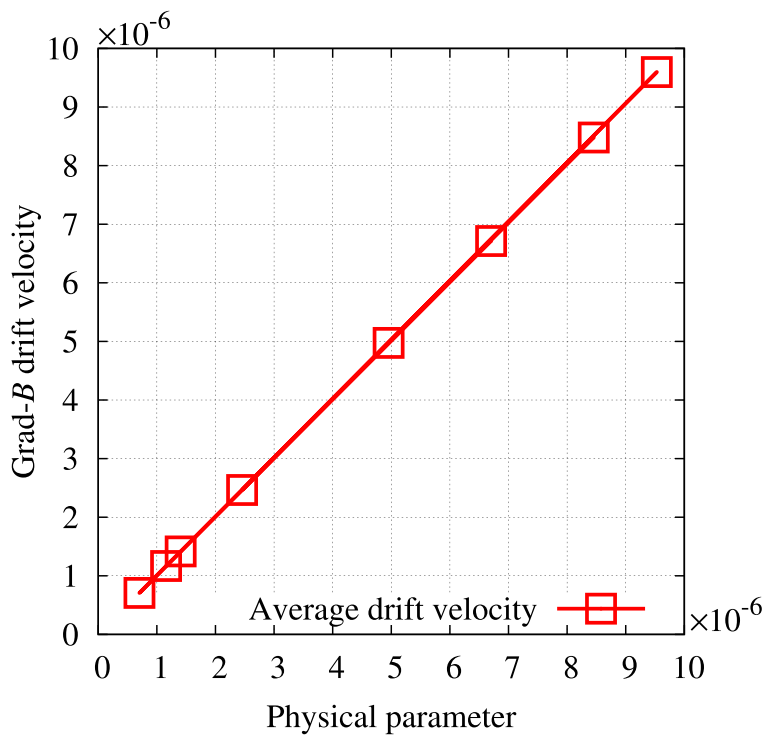

FIG. 2. Grad-B drift velocity $\left\langle\hat{u}_{\nabla B}\right\rangle$ against physical parameter $\left(m v_{0}^{2} / 2 q B L_{B}+3 \hbar / 4 m L_{B}\right)$ with different sets of parameters of charge $q$, magnetic field $B$, mass $m$, initial velocity $\boldsymbol{v}_{0}$, and gradient scale length $L_{B}$.

analytical solutions have good agreement with theoretical analysis, with $0.44 \%$ error. This error is due to the limitation of the analytical grad- $B$ drift velocity operator being solved to the first order in magnetic gradient scale length $L_{B}^{-1}$.

\section{SUMMARY}

In the presence of a weakly non-uniform magnetic field or Landau gauge-like quadratic vector potential of $\boldsymbol{A}=-B \hat{y}\left(1-\hat{y} / 2 L_{B}\right) \boldsymbol{e}_{x}$, a charged particle, i.e., a proton, will encounter drift effect. In this paper, grad- $B$ drift velocity of a charged particle is solved with considering the quantum mechanical effect by using the Heisenberg equation of motion. It is shown that the grad- $B$ drift velocity operator obtained in this study agrees with the classical counterpart when the uncertainty is ignored. The time evolution of the position and momentum operators are also analytically obtained for the non-relativistic spinless charged particle.

The theoretical derivation solutions do agree with the numerical results on the grad- $B$ drift velocity in the presence of a non-uniform magnetic field. The quantum mechanical grad- $B$ drift velocity formulations clearly show the drift velocity dependence on mass $m$ and gradient scale length $L_{B}$. The result implies that light particles with low energy would drift faster than classical drift theory predicts.

Here, the analytical solution of the operator in position is obtained. Thus, it is possible to derive the operator for uncertainty in position, and we are able to analyze the quantum mechanical expansion of the particle. In a future paper, the theoretical derivation on the quantum mechanical expansion of variance of a particle in an inhomogeneous magnetic field will be conducted.

\section{ACKNOWLEDGMENTS}

The authors would like to thank Professor S. Tomioka, Professor Y. Matsumoto, and Professor Emeritus M. Itagaki for their fruitful discussions on the subject. Part of the SOR coding for a GPU was done by Dr. R. Ueda. This research was partially supported by a Grant-in-Aid for Scientific Research (C), Japan, 21560061.

${ }^{1}$ H. Alfvén, "On the motion of a charged particle in a magnetic field," Arkiv Mat. Astr. Fysik 27A, 22 (1940).

${ }^{2}$ L. Spitzer, Physics of Fully Ionized Gases, 1st ed. (Interscience, New York, 1956), pp. 6-7; L. Spitzer, ibid., 2nd ed. (Interscience, New York, 1962) p. 6.

${ }^{3} \mathrm{P}$. W. Seymour, "Drift of a charged particle in a magnetic field of constant gradient," Aust. J. Phys. 12, 309 (1959).

${ }^{4} \mathrm{P}$. W. Seymour, "Exact drift velocity solutions for charged particle motion in a static axially symmetric magnetic field," Aust. J. Phys. 23, 133 (1970).

${ }^{5} \mathrm{~J}$. Hurley, "Drift velocity of a charged particle in an inhomogeneous magnetic field," J. Appl. Phys. 32, 2368 (1961).

${ }^{6} \mathrm{E}$. T. Karlson, "Motion of charged particles in an inhomogeneous magnetic field," Phys. Fluids 5, 476 (1962).

${ }^{7}$ T. J. Birmingham, "Guiding center drifts in time-dependent meridional magnetic fields," Phys. Fluids 11, 2749 (1968).

${ }^{8} \mathrm{H}$. Gelman, "Drift velocities in a time-dependent electric field," Phys. Fluids 13, 1403 (1970).

${ }^{9}$ G. Busoni and G. Frosali, "Asymptotic scattering operators and drift velocity in charged particle transport problems," J. Math. Phys. 34, 4668 (1993).

${ }^{10}$ V. Gavrishchaka, M. E. Koepke, and G. Ganguli, "Dispersive properties of a magnetized plasma with a field-aligned drift and inhomogeneous transverse flow," Phys. Plasmas 3, 3091 (1996).

${ }^{11}$ T. D. Rognlien, D. D. Ryutov, N. Mattor, and G. D. Porter, "Two-dimensional electric fields and drifts near the magnetic separatrix in divertor tokamaks," Phys. Plasmas 6, 1851 (1999).

${ }^{12}$ V. V. Nemov, S. V. Kasilov, W. Kernbichler, and G. O. Leitold, "The $\nabla B$ drift velocity of trapped particles in stellarators," Phys. Plasmas $\mathbf{1 2}$ 112507 (2005).

${ }^{13}$ J. M. Dewhurst, B. Hnat, and R. O. Dendy, "The effects of nonuniform magnetic field strength on density flux and test particle transport in drift wave turbulence," J. Phys. Plasmas 16, 072306 (2009).

${ }^{14} \mathrm{M}$. Yang and M. Ripoll, "Drift velocity in non-isothermal inhomogeneous systems," J. Chem. Phys. 136, 204508 (2012).

${ }^{15} \mathrm{~F}$. Haas, "A magnetohydrodynamic model for quantum plasmas," Phys. Plasmas 12, 062117 (2005).

${ }^{16}$ G. A. Hoshoudy, "Quantum effects on Rayleigh-Taylor instability in a vertical inhomogeneous rotating plasma," Phys. Plasmas 16, 024501 (2009).

${ }^{17}$ D.-H. Ki and Y.-D. Jung, "Magnetic field and quantum screening effects on the occurrence time advance in quantum magnetoplasmas," Phys. Plasmas 18, 074502 (2011).

${ }^{18}$ M. Akbari-Moghanjoughi, "Quantum collapse in ground-state FermiDirac-Landau plasmas,” Phys. Plasmas 18, 082706 (2011).

${ }^{19}$ A. Hasanbeigi, N. Saberi, and H. Mehdian, "Filamentation instability of quantum magnetized plasma in the presence of an external periodic magnetic field," Phys. Plasmas 19, 042112 (2012).

${ }^{20} Y$. Wang, X. Lü, and B. Eliasson, "Modulational instability of spin modified quantum magnetosonic waves in Fermi-Dirac-Pauli plasmas," J. Phys. Plasmas 20, 112115 (2013).

${ }^{21}$ R. E. Marshak, "The radiative and conductive opacities under white dwarf conditions," Ann. N. Y. Acad. Sci. 41, 49 (1941).

${ }^{22}$ R. S. Cohen, L. Spitzer, Jr., and P. McR. Routly, "The electrical conductivity of an ionized gas," Phys. Rev. 80, 230 (1950).

${ }^{23}$ S. Oikawa, T. Oiwa, and T. Shimazaki, "Quantum mechanical plasma scattering," Plasma Fusion Res. 5, S2024 (2010).

${ }^{24}$ S. Oikawa, T. Shimazaki, and T. Oiwa, "Preliminary study on uncertainty driven plasma diffusion II,” Plasma Fusion Res. 5, S2025 (2010).

${ }^{25}$ S. Oikawa, T. Oiwa, and T. Shimazaki, "Preliminary study of uncertaintydriven plasma diffusion," Plasma Fusion Res. 5, S1050 (2010).

${ }^{26}$ L. D. Landau and E. M. Lifshitz, Quantum Mechanics: Nonrelativistic Theory, 3rd ed., translated from the Russian by J. B. Sykes and J. S. Bell (Pergamon Press, Oxford, 1977).

${ }^{27}$ S. I. Braginskii, in Review of Plasma Physics, edited by M. A. Leontovich (Consultants Bureau, New York, 1965).

${ }^{28}$ R. J. Hawryluk, "Results from deuterium-tritium tokamak confinement experiments,” Rev. Mod. Phys. 70, 537 (1998). 
${ }^{29} \mathrm{P}$. K. Chan, S. Oikawa, and E. Okubo, "Numerical analysis of quantum mechanical grad- $B$ drift II," Plasma Fusion Res. 7, 2401034 (2012).

${ }^{30}$ S. Oikawa, P. K. Chan, and E. Okubo, "Numerical analysis of quantum mechanical grad-B drift III," Plasma Fusion Res. 8, 2401142 (2013).

${ }^{31}$ S. Oikawa, W. Kosaka, and P. K. Chan, "Numerical analysis of quantummechanical non-uniform $\boldsymbol{E} \times \boldsymbol{B}$ drift," Plasma Fusion Res. 9, 3401033 (2014).
${ }^{32}$ S. Oikawa, T. Shimazaki, and E. Okubo, "Numerical analysis of quantum mechanical grad- $B$ drift," Plasma Fusion Res. 6, 2401058 (2011).

${ }^{33}$ S. Oikawa, E. Okubo, and P. K. Chan, "Numerical analysis of Schrödinger equation for a magnetized particle in the presence of a field particle," Plasma Fusion Res. 7, 2401106 (2012).

${ }^{34}$ J. Nickolls, I. Buck, M. Garland, and K. Skadron, "Scalable parallel programming with CUDA," ACM Queue 6(2), 40-53 (2008). 